\title{
Research Article \\ Referral Practices for Cognitive Behavioral Therapy for Insomnia: A Survey Study
}

\author{
Deirdre A. Conroy ${ }^{1}$ and Matthew R. Ebben ${ }^{2}$ \\ ${ }^{1}$ Department of Psychiatry, University of Michigan Hospitals and Health Systems, Ann Arbor, MI 48109, USA \\ ${ }^{2}$ Center for Sleep Medicine, Weill Medical College, Cornell University, New York, NY 10065, USA \\ Correspondence should be addressed to Deirdre A. Conroy; daconroy@umich.edu
}

Received 12 March 2015; Revised 5 July 2015; Accepted 7 July 2015

Academic Editor: Andrea Romigi

Copyright ( 2015 D. A. Conroy and M. R. Ebben. This is an open access article distributed under the Creative Commons Attribution License, which permits unrestricted use, distribution, and reproduction in any medium, provided the original work is properly cited.

\begin{abstract}
This study examined referring practices for cognitive behavioral therapy for insomnia (CBTI) by physicians at University of Michigan Hospitals and Weill Cornell Medical College of Cornell University. A five-item questionnaire was sent via email that inquired about the physician's patient load, number of patients complaining of insomnia, percent referred for CBTI, and impressions of what is the most effective method for improving sleep quality in their patients with insomnia. The questionnaire was completed by 239 physicians. More physicians believed a treatment other than CBTI and/or medication was most effective $(N=83)$. "Sleep hygiene" was recommended by a third of the sample. The smallest number of physicians felt that CBTI alone was the most effective treatment $(N=22)$. Additional physician education is needed.
\end{abstract}

\section{Introduction}

Insomnia is one of the most common health complaints. Population prevalence estimates vary between $9 \%$ [1] and 57\% in older individuals [2]. In the recent past, chronic insomnia was almost always viewed as a symptom of medical and mental disorders [3]. In this conceptualization, it made sense to focus on the evaluation and treatment of the underlying causes of trouble sleeping. The new conceptualization of insomnia, as codified in the DSM V [4] and ICSD 3 [5], acknowledges insomnia's independent status as a condition warranting clinical attention. The implications of this new view are that clinicians should not wait until they have addressed the medical and psychiatric comorbidities before they intervene in sleep disturbance.

The recent evidence of the effects of insomnia on medical and mental disorders has helped establish the reciprocal or bidirectional relationship between insomnia and medical and mental disorders [6-9]. Furthermore, the development of effective nonpharmacological treatments, such as cognitive behavioral treatment for insomnia (CBTI), that demonstrates effectiveness in insomnia with and without a wide range of comorbidities has given physicians and sleep specialists a larger set of therapeutic options compared to what were available in the past [10-13]. Taken together, the understanding that insomnia is not just a symptom of other disorders, nonpharmacological treatments are as effective as medications [14], and insomnia is common predicts that physicians can be expected to rely more on specialists who are adept at treating sleep disturbance. However, there is a mismatch between the number of patients with insomnia and those receiving CBTI treatment. The issue of access to clinicians with expertise in delivering this therapy has been raised as one of the reasons accounting for this mismatch [15]. However, even in clinical practices where these services are available, clinicians with CBTI training may not experience the high patient volume one might expect. This may be due, in part, to patients' preference for a quick fix for insomnia with medications [16]. Another hypothesis is that physicians may not be aware of CBTI as a viable treatment option for their patients or they may not be knowledgeable about the therapy, despite its widespread use. Moreover, two recent studies highlight the importance of using CBTI as the primary tool to treat insomnia $[17,18]$. Both studies found an increase in mortality associated with the use of hypnotic medication. 
TABLE 1: Referral practices for insomnia treatment and patient volume by physician specialty.

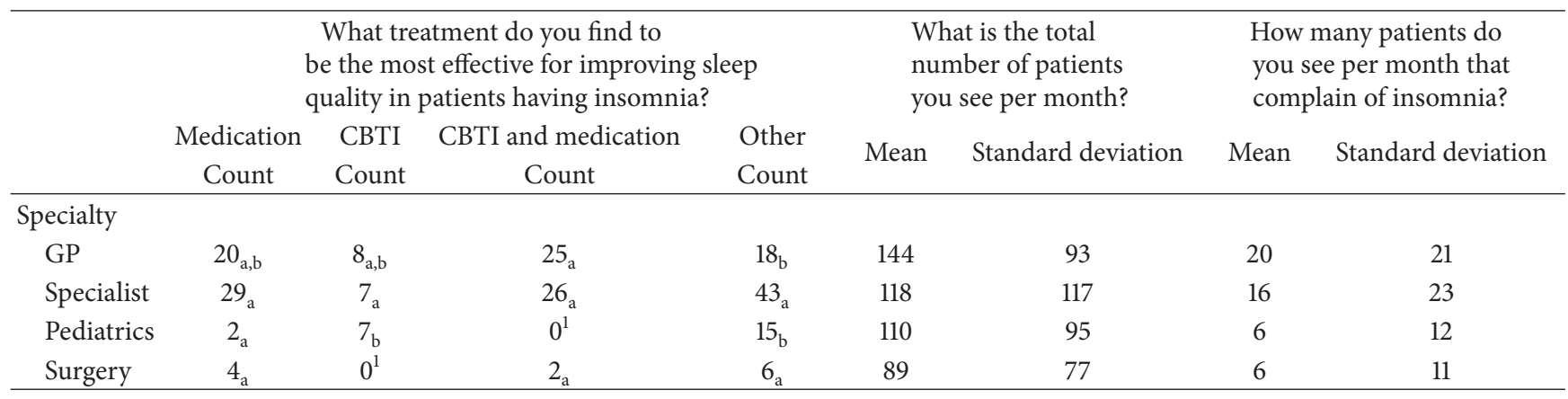

Note: values in the same row and subtable not sharing the same subscript are significantly different at $p<0.05$ in the two-sided test of equality for column proportions. Cells with no subscript are not included in the test. Tests assume equal variances. ${ }^{2}$

${ }^{1}$ This category is not used in comparisons because its column proportion is equal to zero or one.

${ }^{2}$ Tests are adjusted for all pairwise comparisons within a row of each innermost subtable using the Bonferroni correction.

The purpose of this study was to investigate the referral practices and attitudes of physicians towards insomnia treatment at two academic medical centers in the United States. We sought to examine physician's opinions of treatment efficacy of medication and/or CBTI for the treatment of insomnia. This information may be useful in determining a strategy to educate medical providers on effective nonpharmacological treatment options for patients with insomnia.

\section{Methods}

2.1. Participants. In order to obtain a diverse sample, we selected two large area hospitals in the Midwest and Eastern United States. Weill Cornell Medical Center is part of New York-Presbyterian University Hospital of Columbia and Cornell and is located in Manhattan, NY. It is a 2,264-bed general medical and surgical facility with 105,339 admissions in the most recent year reported. It performed 30,739 annual inpatient and 76,689 outpatient surgeries. New York-Presbyterian University Hospital of Columbia and Cornell is a teaching hospital. Weill Cornell Medical Center has two clinical psychologists in faculty at the Center for Sleep Medicine that are certified in behavioral sleep medicine. The University of Michigan Hospitals and Health Centers is located in Ann Arbor, MI. It is a 919-bed general medical and surgical facility with 45,137 admissions in the most recent year reported. It performed 17,784 annual inpatient and 28,270 outpatient surgeries [19]. It is also a teaching hospital. There are three clinical psychologists in faculty in the Behavioral Sleep Medicine Clinic at the University of Michigan Hospitals and Health Systems that are certified in behavioral sleep medicine.

2.2. Questionnaire Survey. A five-item questionnaire was created by the authors to illicit information about clinical specialty, patient flow, and referral practices for CBTI. The items included the following. (1) What is your primary specialty? (2) What is the total number of patients you see per month? (3) How many patients do you see per month that complain of insomnia? (4) What percentage of your patients do you refer for cognitive behavioral therapy for insomnia (CBTI)? (5) What treatment do you find is the most effective for improving sleep quality in patients with insomnia: (A) medication, (B) CBTI, (C) a combination of CBTI and medication, and (D) other? A text response could then be entered. The questionnaire was conducted with Survey Monkey, internet based survey software.

An email with the link to the survey was sent through an email that reached the medical staff at the University of Michigan Hospitals and Health Systems $(n=1600)$ and New York Presbyterian Hospital-Cornell Medical Center $(n=1511)$. Respondents completed the survey by clicking a link to the survey website included in the email. The project was exempted by both the University of Michigan (HUM00079350) and Weill Cornell Medical College (1308014173) Institutional Review Boards.

\section{Data Analysis}

Quantitative data was analyzed using SPSS version 22 (IBM Software, Armonk, NY). A one-sample chi-square test was conducted to assess differences in proportions. Equal frequencies were expected among responses to questions. Qualitative analysis was used to evaluate free text responses. This was performed with the Text Analysis tool provided by Survey Monkey. All terms yielding response rates greater than $10 \%$ are described in Results. Terms used in less than $10 \%$ of responses are listed only if they could have been viewed as belonging to the "medication" or "CBTI" answers to question 5 of the survey.

\section{Results}

A total of 239 clinicians responded to the survey. Most respondents considered themselves either internal/family medicine physicians $(n=57,24.3 \%)$ or "other" $(n=50$, $21.3 \%)$. Other common specialties included pediatrics $(n=$ $28,11.9 \%)$, psychiatry $(n=17,7.2 \%)$, surgery $(n=14,6.0 \%)$, and oncology $(n=11,4.7 \%)$. The remaining specialties included pulmonology (2.1\%), nursing (0.9\%), ENT $(2.6 \%)$, cardiology (3.0\%), psychology (3.4\%), neurology $(2.6 \%)$, urology (0.9\%), OB/GYN (3.0\%), GI (0.9\%), sleep medicine $(1.7 \%)$, or geriatrics $(3.8 \%)$. See Table 1 for a breakdown of prescribing practices by specialty. 


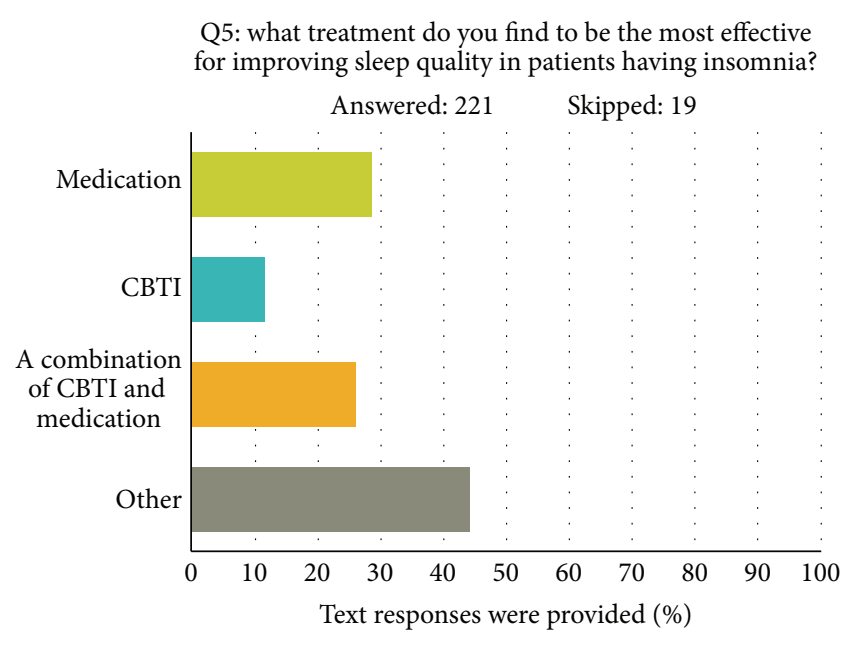

FIGURE 1

Respondents saw an average of $121(\mathrm{SD}=106)$ patients per month (range $0-1000$ ) and an average of 15.2 (SD = 21) patients per month with insomnia ( $12 \%$ patients with insomnia per month). However, the average number of patients referred for CBTI per month was $1.5(\mathrm{SD}=4.9)$. Opinion of treatment efficacy was significantly different among responders to the survey, $X^{2}(3, N=213)=35.02$, $p=0.000$. The effect size was 0.06 .

The number of physicians who believed a treatment other than CBTI and/or medication was most effective $(N=83)$ was much larger than the hypothesized number of 53.3 (see Figure 1). A qualitative analysis of these responses revealed that $29.6 \%$ contained the term "sleep hygiene," and $4 \%$ used the term "relaxation." The word "medication" or "melatonin" was used in $7.1 \%$ of responses. Other commonly used terms included "no experience" (18.4\%), "treat medical condition" (12.2\%), "not applicable" (11.2\%), and "refer to PCP” (11.2\%). The smallest number of physicians felt that CBTI alone was the most effective treatment $(N=22)$. A total of $n=$ 55 and $n=53$ doctors thought that medication and a combination of medication and CBTI, respectively, were the most effective treatment approaches. Other text responses to other treatments included remedies such as "warm milk and hydroxyzine" as treatment for insomnia.

\section{Discussion}

The purpose of this study was to investigate the treatment approaches used for insomnia by medical professionals at two large academic medical centers in the United States. The study shows that although respondents in our survey see many patients per month, very few (approximately 1\%) of these patients are referred for treatment with CBTI alone. This is particularly surprising given that both the University of Michigan and Weill Cornell Medical College have well known sleep laboratories with practitioners certified in behavioral treatments for insomnia. Therefore, the dearth of referrals could not be attributed to a lack of appropriate treatment facilities, which could be the case elsewhere. The responses to "other" for question number 5 "What treatment do you find is the most effective for improving sleep quality in patients with insomnia?" suggest that the term CBTI may not be well understood. Physicians commonly reported recommending "sleep hygiene" and "relaxation techniques" instead of CBTI, even though these are components of the multimodal CBTI approach to treatment. This is consistent with a similar large survey study of 296 general practitioners in England, which revealed that sleep hygiene advice is provided by $88 \%$ of GPs [20]. Moreover, recommending components of CBTI, such as "sleep hygiene" alone, which have not been shown to independently improve sleep quality [21], may lead to poor patient treatment outcomes. This study suggests that although scientifically rigorous research studies have found CBTI effective for insomnia and numerous disorders comorbid with insomnia [22], additional education to the medical community on CBTI is needed. Educational programs may include but are not limited to hospital seminars, grand round lectures, colloquiums, or mentorships of residents or other health care professionals in CBTI. Training programs for nonsleep specialists [23], such as the one utilized by the Veterans Health Administration, provide an example of an effective way to train clinicians in CBTI [24] and improve insomnia in that patient population [25].

One limitation to our study is that the University of Michigan and Weill Cornell Medical College/New YorkPresbyterian Hospitals are tertiary referral hospitals with few primary care doctors. Therefore, other regional medical centers, with a higher overall percentage of primary care practitioners, may have different referral patterns. However, most regional centers do not have behavioral sleep medicine experts, and many of the referrals made to sleep laboratories (most behavioral sleep medicine specialists are affiliated with a sleep laboratory) are made by pulmonologists, ear, nose, and throat specialists, and cardiologists. As a result, we believe that our data provide a fair analysis of the referral network seen by many behavioral sleep medicine experts.

\section{Summary}

CBTI is the first line treatment for insomnia and is now well validated as being effective in the short and long term [26]. A brief survey completed by 239 medical professionals at two large academic medical centers revealed that therapies other than CBTI seem to be most commonly recommended for patients with insomnia. Medical education programs within medical settings to promote proper insomnia treatments are needed.

\section{Conflict of Interests}

The authors declare that there is no conflict of interests regarding the publication of this paper.

\section{Acknowledgment}

The authors would like to thank Susan Chan for her work on this project. 


\section{References}

[1] M. M. Weissman, A. S. Brown, and A. Talati, "Translational epidemiology in psychiatry: linking population to clinical and basic sciences," Archives of General Psychiatry, vol. 68, no. 6, pp. 600-608, 2011.

[2] M. Godard, Z. Barrou, and M. Verny, "Geriatric approach of sleep disorders in the elderly," Psychologie et NeuroPsychiatrie $d u$ Vieillissement, vol. 8, no. 4, pp. 235-241, 2010.

[3] M. M. Weissman, P. Wickramaratne, Y. Nomura, V. Warner, D. Pilowsky, and H. Verdeli, "Offspring of depressed parents: 20 years later," American Journal of Psychiatry, vol. 163, no. 6, pp. 1001-1008, 2006.

[4] American Psychiatric Association, Diagnostic and Statistical Manual of Mental Disorders, American Psychiatric Publishing, Arlington, Va, USA, 5th edition, 2013.

[5] American Academy of Sleep Medicine, International Classification of Sleep Disorders, American Academy of Sleep Medicine, Darien, Ill, USA, 3rd edition, 2014.

[6] M. H. Bonnet, G. G. Burton, and D. L. Arand, "Physiological and medical findings in insomnia: implications for diagnosis andcare," Sleep Medicine Reviews, vol. 18, no. 2, pp. 111-122, 2014.

[7] S. Goldman-Mellor, A. M. Gregory, A. Caspi et al., "Mental health antecedents of early midlife insomnia: evidence from a four-decade longitudinal study," Sleep, vol. 37, no. 11, pp. 17671775, 2014.

[8] A. Hayley, L. J. Williams, K. Venugopal, G. A. Kennedy, M. Berk, and J. A. Pasco, "The relationships between insomnia, sleep apnoea and depression: findings from the American National Health and Nutrition Examination Survey, 20052008," Australian \& New Zealand Journal of Psychiatry, vol. 49, no. 2, pp. 156-170, 2015.

[9] J. A. Shekleton, E. E. Flynn-Evans, B. Miller et al., "Neurobehavioral performance impairment in insomnia: relationships with self-reported sleep and daytime functioning," Sleep, vol. 37, no. 1, pp. 107-116, 2014.

[10] C. A. Espie, “'Stepped care': a health technology solution for delivering cognitive behavioral therapy as a first line insomnia treatment," Sleep, vol. 32, no. 12, pp. 1549-1558, 2009.

[11] S. M. McCurry, R. G. Logsdon, L. Teri, and M. V. Vitiello, "Evidence-based psychological treatments for insomnia in older adults," Psychology and Aging, vol. 22, no. 1, pp. 18-27, 2007.

[12] C. M. Morin, R. R. Bootzin, D. J. Buysse, J. D. Edinger, C. A. Espie, and K. L. Lichstein, "Psychological and behavioral treatment of insomnia: update of the recent evidence (19982004)," Sleep, vol. 29, no. 11, pp. 1398-1414, 2006.

[13] A. Siebern and R. Manber, "New developments in cognitive behavioral therapy as the first-line treatment of insomnia," Psychology Research and Behavior Management, vol. 4, pp. 2128, 2011.

[14] C. M. Morin, J. P. Culbert, and S. M. Schwartz, "Nonpharmacological interventions for insomnia: a meta-analysis of treatment efficacy," American Journal of Psychiatry, vol. 151, no. 8, pp. 11721180, 1994.

[15] W. R. Pigeon, V. M. Crabtree, and M. R. Scherer, "The future of behavioral sleep medicine," Journal of Clinical Sleep Medicine, vol. 3, no. 1, pp. 73-79, 2007.

[16] M. E. Moloney, R. Konrad, and C. R. Zimmer, "The medicalization of sleeplessness: a public health concern," American Journal of Public Health, vol. 101, no. 8, pp. 1429-1433, 2011.

[17] P. M. Lewinsohn, R. E. Roberts, J. R. Seeley, P. Rohde, I. H. Gotlib, and H. Hops, "Adolescent psychopathology. II.
Psychosocial risk factors for depression," Journal of Abnormal Psychology, vol. 103, no. 2, pp. 302-315, 1994.

[18] B. Sivertsen, A. G. Harvey, A. J. Lundervold, and M. Hysing, "Sleep problems and depression in adolescence: results from a large population-based study of Norwegian adolescents aged 16-18 years," European Child and Adolescent Psychiatry, vol. 23, no. 8, pp. 681-689, 2014.

[19] U.S.News \& World Report LP Health Hospitals Report, 2014.

[20] H. Everitt, L. McDermott, G. Leydon, H. Yules, D. Baldwin, and P. Little, "GPs' management strategies for patients with insomnia: a survey and qualitative interview study," British Journal of General Practice, vol. 64, no. 619, pp. el12-e119, 2014.

[21] T. Morgenthaler, M. Kramer, C. Alessi et al., "Practice parameters for the psychological and behavioral treatment for insomnia: an update. An American Academy of Sleep Medicine Report," Sleep, vol. 29, no. 11, pp. 1415-1419, 2006.

[22] J. Williams, A. Roth, K. Vatthauer, and C. S. McCrae, "Cognitive behavioral treatment of insomnia," Chest, vol. 143, no. 2, pp. 554-565, 2013.

[23] R. Manber, C. Carney, J. Edinger et al., "Dissemination of CBTI to the non-sleep specialist: protocol development and training issues," Journal of Clinical Sleep Medicine, vol. 8, no. 2, pp. 209218,2012

[24] M. Trockel, B. E. Karlin, C. B. Taylor, and R. Manber, "Cognitive Behavioral Therapy for insomnia with veterans: evaluation of effectiveness and correlates of treatment outcomes," Behaviour Research and Therapy, vol. 53, no. 1, pp. 41-46, 2014.

[25] B. E. Karlin, M. Trockel, A. P. Spira, C. B. Taylor, and R. Manber, "National evaluation of the effectiveness of cognitive behavioral therapy for insomnia among older versus younger veterans," International Journal of Geriatric Psychiatry, vol. 30, no. 3, pp. 308-315, 2015.

[26] C. M. Morin, C. Colecchi, J. Stone, R. Sood, and D. Brink, "Behavioral and pharmacological therapies for late-life insomnia: a randomized controlled trial," Journal of the American Medical Association, vol. 281, no. 11, pp. 991-999, 1999. 


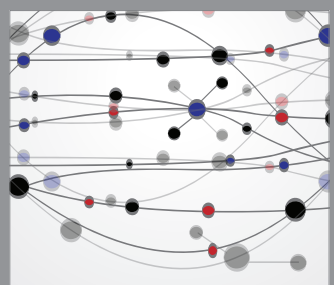

The Scientific World Journal
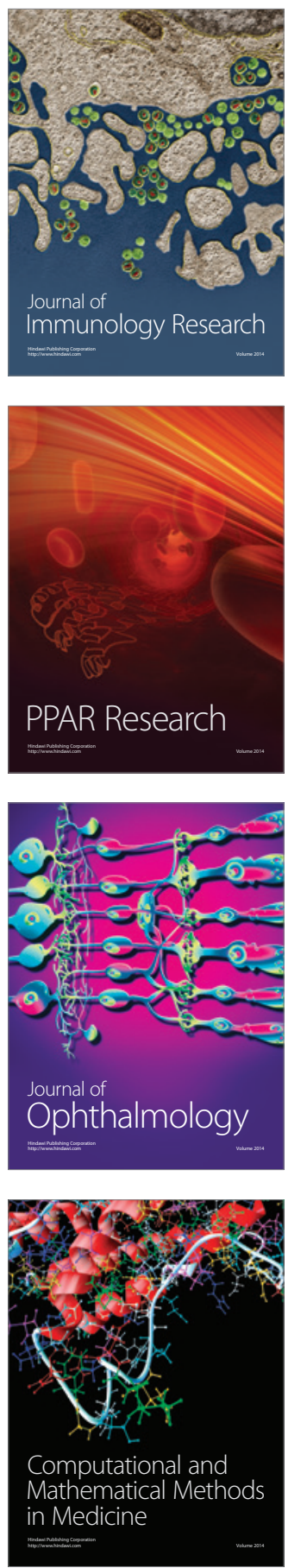

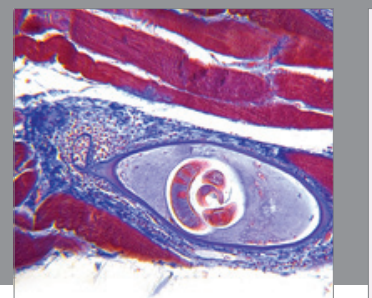

Gastroenterology

Research and Practice
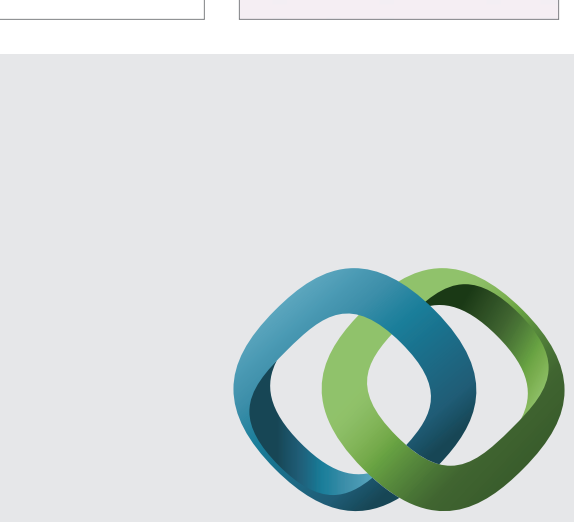

\section{Hindawi}

Submit your manuscripts at

http://www.hindawi.com
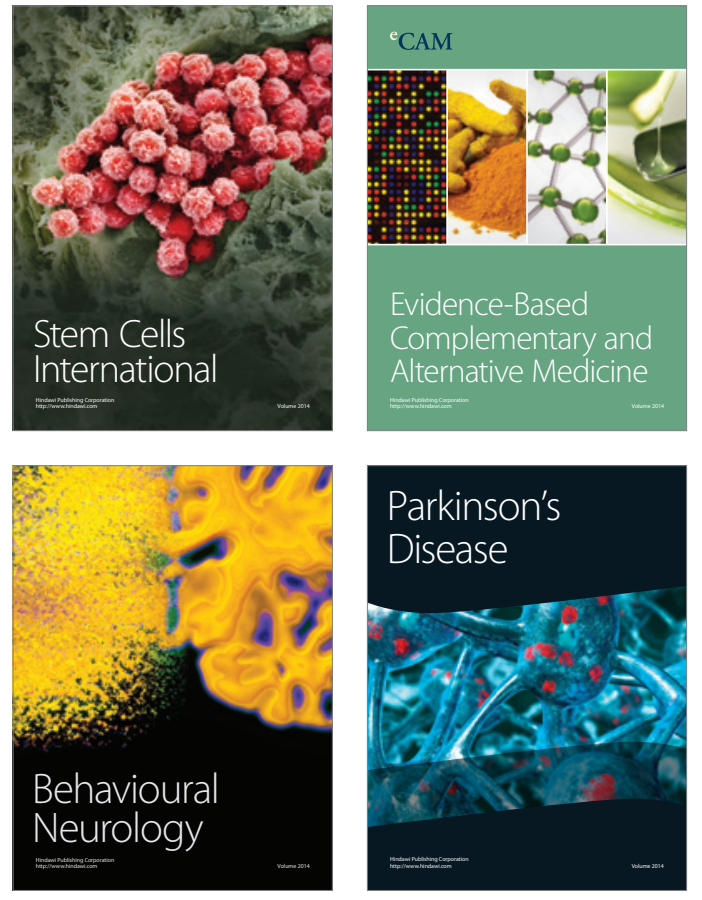
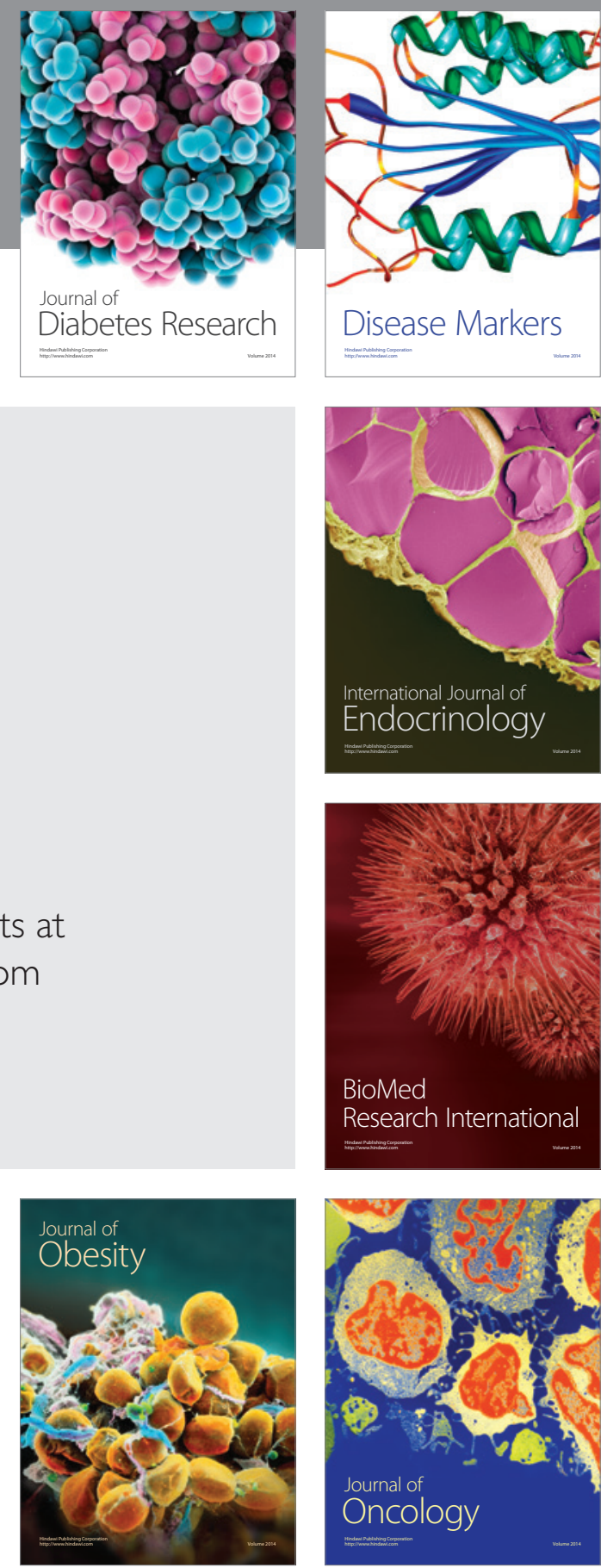

Disease Markers
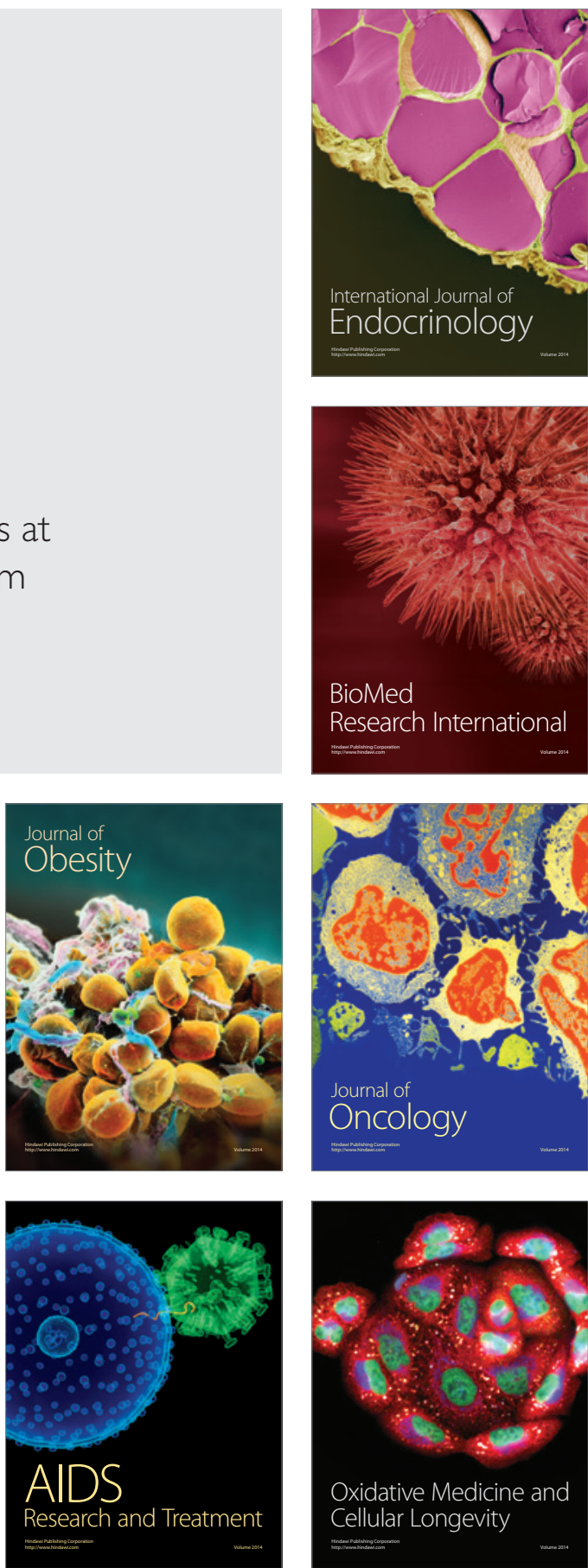